\title{
QuesTInSitu: from tests to routes for assessment in situ activities
}

\author{
Patricia Santos*, Mar Pérez-Sanagustín, Davinia Hernández-Leo, Josep Blat \\ Department of Information and Communication Technologies, Universitat Pompeu Fabra, Barcelona, Spain \\ \{patricia.santos, mar.perez, davinia.hernandez, josep.blat\}@upf.edu
}

\begin{abstract}
Test-based assessment tools are mostly focused on the use of computers. However, advanced Information and Communication Technologies, such as handheld devices, opens up the possibilities of creating new assessment scenarios, increasing the teachers' choices to design more appropriate tests for their subject areas. In this paper we use the term Computing-Based Testing (CBT) instead of Computer-Based Testing, as it captures better the emerging trends. Within the CBT context, the paper is centred on proposing an approach for "Assessment in situ" activities, where questions have to be answered in front of a real space/location (situ). In particular, we present the QuesTInSitu software implementation that includes both an editor and a player based on the IMS Question and Test Interoperability specification and GoogleMaps. With QuesTInSitu teachers can create geolocated questions and tests (routes), and students can answer the tests using mobile devices with GPS when following a route. Three illustrating scenarios and the results from the implementation of one of them in a real educational situation show that QuesTInSitu enables the creation of innovative, enriched and context-aware assessment activities. The results also indicate that the use of mobile devices and location-based systems in assessment activities facilitates students to put explorative and spatial skills into practice and fosters their motivation, reflection and personal observation.
\end{abstract}

\section{Keywords}

improving classroom teaching; interactive learning environments; mobile assessment; assessment in situ

\section{Introduction}

Objective tests are used in Computer-Based Testing to assess knowledge in scenarios where users have to response questions whose correct answers are predetermined (Bull \& McKenna, 2004). Typically, these tests are administered in large computing labs in large enrolment courses. Their main aim is to help teachers in grading students and to provide students with automatic feedback. Recently, different ubiquitous computing devices have begun to be used in assessment, such as mobile phones or PDAs. The term mobile-learning (m-learning) refers to the use of these devices in education (Sharples, Corlett, \& Westmancott, 2002). With mobile devices students can answer tests and practice self-assessment anytime and anywhere (Chen, 2010; de-Marcos et al., 2010; Question Mark, 2010; Triantafillou, Georgiadou, \& Economides, 2008; Zhang, Wills, Gilbert, Warburton, \& Bacigalupo, 2010). However, we claim that the majority of mobile test (m-test) approaches are not exploiting all the possibilities of the anywhere concept. The features of a mobile phone can be used to design innovative types of assessment activities to be performed outside the classroom.

Within this context, in this paper we propose, on the one hand, a new type of assessment activity based on test, called Assessment in situ, where users have to use a mobile phone to follow and answer a testroute putting context-aware skills into practice; and, on the other hand, we present a system, called QuesTInSitu, that enables the creation and enactment of these routes. QuesTInSitu allows teachers to create activities based on tests and mediated by computers and smart-phones. Each question has associated a geographical coordinate, what makes the questions context-dependent. This means that a user has to be positioned in a correct place in order to get the questions displayed. The questions created with QuesTInSitu are compliant with the educational technology standard for assessment IMS Question \& Test Interoperability (QTI) (IMS, 2006). Educational technology standards for assessment are used for supporting interoperability and re-usability of assessment resources (Miao, Tattersall, Schoonenboom, Stevanov, \& Aleksieva-Petrova, 2007). QTI is the facto standard used for computationally representing questions and tests. The specification provides an interoperable data model for the representation of questions (items), tests, their outcomes and feedback (Harchay, Cheniti, \& Braham, 2010). This interoperability enables the exchange of assessment resources between QTI-compliant systems which include an engine that interpret the computationally represented tests.

QuesTInSitu integrates an assessment engine as a module that is used to interpret the QTI questions and test that are created with the authoring section of the system. QuesTInSitu is not extending QTI with new types of questions. It uses traditional QTI questions such as Multiple Choice, Multiple Response and 
Yes/No questions. The contribution deals with using traditional type of questions with the added value that the questions have to be answered in situ using a mobile device. Then, an open space, like a city, is used as a canvas where questions are located and organized. The information of this space has to be used by the students, to reflect about the questions and do the necessary actions to answer them correctly. Teachers can use the elements of the physical space (buildings, monuments, plants, people, among others things) with assessment purposes without going physically to tag the elements.

With the aim of demonstrating the educational benefits and possibilities of using QuesTInSitu for designing assessment in situ activities, we present and analyse three scenarios: (1) An experiment was conducted with a group of students and teachers during the realization of a real assessment in situ activity. It was carried out in an authentic educational context. The participants were students and teachers of the second course of a humanistic High School in Catalonia. Six tests (which correspond to six different routes by districts in the city of Barcelona) were created with QuesTInSitu. The students had to answer QTI questions that appear in their mobile when they were in specific pre-determined positions. (2) An enhancement of a third party scenario that exemplifies how QuesTInSitu can be used to improve and expand an authentic ubiquitous learning activity mediated by mobiles devices. The case describes the benefits of combining the Mobile Plant Learning System (MPLS), proposed by Huang Y. et al (2009) for learning botany, with QuesTInSitu. The combination enriches the activity with the introduction of questions in situ so as to enable the assessment of students' knowledge and skills while they are observing the plants. Finally, (3) the last scenario illustrates how QuesTInSitu facilitates the edition and execution of existing cultural itineraries with assessment purposes.

The scenarios exemplify how assessment in situ activities can be done using geolocated questions with smartphones and GPS data. However, other technologies like NFC tags or QR-Codes could be also used to create assessment in situ activities. After presenting the scenarios, the paper discusses the advantages and limitations (for teachers and students) of using GPS information versus other methods.

The remainder of the paper is organized as follows. In Section 2 we present a brief review and discussion of the state of the art in ICT applied to Assessment, m-learning and assessment in situ activities that motivate the aims of this research. Section 3 introduces the QuesTInSitu system while Section 4 presents the main scenario evaluated in an experiment with real students and teachers. Section 5 includes two examples of scenarios to illustrate the applicability of QuesTInSitu in diverse educational disciplined. Section 6 is focused on discussing the results of the evaluation and the use of different technologies to create assessment in situ activities. Finally, Section 7 includes the conclusions and the future work derived from the contributions of this paper.

\section{Background \& motivation}

The use of ICT devices and implementations make possible the creation of new types of assessment activities based on tests. Focusing our attention on the use of smart-phones with educational purposes, the features of these devices enable the design of activities outside the classroom. This section introduces and discusses the advantages of using mobile devices for assessing anywhere.

\subsection{Computing-Based Testing: the evolution of Computer-Based Testing}

As Bull \& McKenna (2004) shown in their blueprint for Computer Assisted Assessment, the use of objective tests in education is probably more widespread than most people realise. As we have indicated in the Introduction, until recently computers have been the unique devices used to play objective tests. Most of researchers in this field have concentrated their efforts in creating innovative question-items or using complex computational solutions (such as video games) to represent tests. They claim that the creation of new types of questions can improve the measuring of students' knowledge and skills. As has been studied by researchers, computers can be used to assess higher order skills that are impossible or very difficult to assess using traditional methods (Boyle \& Hutchison, 2009; Parshall, Harmes, Davey, \& Pashley, 2010; Ridgway \& McCusker, 2003).

We agree with these researchers that the use of computers is beneficial for assessment and allow teachers to create new types of questions and tests on the assessment of higher order skills. However, we also think that traditional types of questions (such as Multiple Choice, Multiple Response or Yes and No) can still be used to create enriched and innovative activities for assessing complex skills. Although these new activities may not innovate in terms of type of types of questions they can innovate in terms of technology. For instance, the use of mobile technologies with educational purposes has been identified as beneficial for encouraging the development of meta-level thinking skills (Wegerif, 2002; Facer et al., 2004). Elliot (2008), in his paper called “Assessment 2.0", claims that it is necessary to explore new ways 
of modernising assessment in order to be closer to today's learners. He proposes that the solution is to use Web 2.0 assessment tools, because its use can enhance students' engagement. As Elliot, we state that it is necessary to innovate in assessment in order to create adequate solutions for the current digital natives' students. But apart from computers (and Web 2.0), other technologies, such as mobile phones or PDAs, are also close to students and are having a growing impact in the field of assessment based on tests (Dearnley et al., 2009).

Considering the points discussed in this section, we claim that nowadays the term Computer-Based Testing (CBT) does not capture all the technological possibilities of representing objective tests. For this reason we propose to use an evolution of the term that is: Computing-Based Testing (also CBT). The word computing covers the different ways of technologically representing and manipulating a test. In this paper we use the term CBT, where the "C" stands for "Computing". Specifically we focus our attention on the use of smartphones and ubiquitous-learning systems for doing an innovative assessment activity based on test outside a classroom.

\subsection{Smartphones and Computing-Based Testing}

The broad acceptance of mobile technology has led to a new educational paradigm known as mobilelearning (m-learning). As Jones \& Jo (2004) clarify, m-learning includes the concept of anytime/anywhere in Technology enhanced Learning. As we have introduced, in the field of CBT new test tools have been developed in order to be used in mobile phones or PDAs. We have selected three systems as examples that represent the characteristics and educational uses of common m-test approaches: (1) de-Marcos et al (2010) present an experiment where they evaluate their own implementation for practicing online self-assessment test activities using mobile phones. They measure the improvement of achievement of knowledge with groups of three different courses. Their objective is to use the system to support traditional class-based learning. The study demonstrates that thanks to the motivation that those students showed using mobile phones to practise self-assessment activities, the students' achievement improved. (2) Zhang et al (2010) have implemented a QTI engine for supporting offline m-learning assessment. The system allows users to practice self-assessment having in local the tests that they want to practice.(3) Triantafillou et al (2008)evaluate a Computerized Adaptive Test (CAT) prototype for mobile devices. The system adapts the questions taking into account the answers of the user. Their objective is to design a CAT solution that can be used anytime and anywhere, emphasizing again that students could use the solution to enhance their knowledge practicing self-assessment.

The approaches presented are good representative examples that illustrate common m-test tools. The first two use the QTI standard for assessment. As the authors explain in their papers, this standard is used to model the assessment resources and facilitate the interoperability between systems (of any type of device). The three studies conclude saying that the use of mobiles can enhance the field of CBT thanks to the fact that students can answer the test "anytime and anywhere”. Also, they find that the use of mobiles motivates the students to practice self-assessment. However, none of these proposals is innovating in terms of assessing higher order skills. The added value of these solutions is basically focused on enabling students to answer tests wherever and whenever they want.

Current mobile phones integrate features such as cameras, Internet connection, GPS, etc. Several researchers study the effect of using these features to support educational activities. For instance, Sharples et al. (2002) present a study which explains how to support students to capture images, notes and sounds with their mobiles phones so that they can relate these data with existing web-based learning resources or share them with their class-mates or teachers. Facer et al. (2004) explain how mobile technologies can be combined with principles of self-motivation and engagement to create an exploratory learning experience that support students in their interaction with the space and with other players. Huang, Lin, \& Cheng (2009) describe how the GPS and the camera of a mobile phone can be used to create an innovating activity for learning botany outside the classroom. Finally, Hwang \& Chang (2011) research the use of mobile devices, wireless communication and context awareness to assess in situ the knowledge of students about local culture in Taiwan. They did a control experiment where they compare the answering of questions using a traditional guide-tour application versus a PDA with a Formative Assessment-based Mobile Learning application (FAML). The aim of the experiment was to analyse the effect of providing adaptive methods during the performance of an assessment in situ activity. However, in this case the authors do not exploit the possibilities of location-based systems to geolocalize and automatically deliver the assessment questions in situ.

The previous examples illustrate context-aware activities that consider the space outside the classroom as an adequate location for learning and assessing students'skills. The integration of the GPS in smartphones has been a success and has turned mobile phones into location-based systems (LBS). The LBS feature of mobiles phones has been combined with the augmented reality paradigm to develop 
applications where people use their mobiles to superimposed (or view) digital information to real positions in the world (Mackay, 1998). In this context, it becomes important to identify the purposes of using context-aware mechanisms when designing mobile ubiquitous learning/assessment experiences. Nova, Girardin \& Dillenbourg (2005), performed an experiment where groups of students had to collaborate using Tablet PCs. The game CatchBob! consists in finding a virtual object in a campus by collaborating with team mates. As an experimental condition they studied the effect of using (or not) context-aware mechanisms with different groups. All the groups had the possibility of sending notations to their partners. The groups without context-aware mechanisms had better communication with their partners than the groups that had the awareness. Their conclusion was that "location is not enough": space and location-awareness are interesting information that can be used in ubiquitous experiences but it can not be limited to a simple broadcast of people's positions. An example of authoring tool that enable people to design and play ubiquitous location-based activities using a mobile phone (with GPS and Windows Mobile) is Mscape (Stenton et al., 2007). A specific web site for educators (see http://www.createascape.org.uk/ ) explains how to create learning activities using this tool. An author can associate a learning resource (an HTML, audio, or text) with a real coordinate. Then a route can be created and played through a mobile phone. When users are situated with their mobile phone in a location of the route, an event appears on the mobile screen and the user can view the information of the resource. Until now the educational experiences designed with Mscape have been realised with learning purposes and not with assessment purposes.

Considering the current features that smartphones integrate and the type of activities that have been designed using mobiles with assessment purposes, we state that new types of assessment activities based on test and mediated by mobiles phones can be implemented. According to some of the researchers cited above (Hwang et al., 2011; de-Marcos et al., 2010; Facer et al., 2004) and others studies (Naismith, Lonsdale, Vavoula, \& Sharples, 2004) the use of mobile devices has a positive impact in the students' motivation and learning. The studies agree that these devices engage students' participation in the activity. In this sense, we also think that students need to have some control in assessment activities, encouraging its participation.

\subsection{Assessment in situ activities: answering questions considering the real world environment}

From the previous basis we propose a new type of CBT activity based on m-learning and context-aware learning and refer to it as an Assessment in situ activity. The majority of the m-test solutions have in common that they focus their attention on creating tools for being used anytime and anywhere. In fact they use mobile phones because these devices are place-independent and then the students can use them in their free time (e.g. while they are travelling or at home) to practice self-assessment activities. But these approaches do not exploit mobiles to design new types of assessment activities. This paper focuses on assessment activities that cannot be practiced without mobile devices, and on the contrary to other mtest activities, they are dependent of specific locations. The words in situ mean that questions only can be answered when users are correctly placed in a specific location. Each question is associated with a real geographic coordinate, when the user is in an area near of the coordinate the question can be answered using a smartphone. Students need to be located in situ in the correct place because they will need to interact with the environment (observing, touching, talking with people, etc.) in order to understand the question and answer it. In an assessment in situ activity students put into practice transversal skills such as exploration, spatial and observation besides of the specific skills related with the content of the test. Current m-test tools are student-centered solutions where the focus of the activity is the student and the teacher has a secondary (or invisible) role. In our approach teachers have an important role. They create but also orchestrate the activity, monitoring all the actions of their students in real time.

An assessment in situ activity can be divided into two different types: (1) Assessment in (virtual) situ: the student does not need to be physically in the real position related to the question for answering it; the questions are positioned in a web map through a web based application and students answer the questions using the information that the map contains. In this case students need to be aware of the contextual information of the web map. (2) Assessment in (real) situ: a question is associated to a real physical space. The structure of the activity determines that the questions have to be answered using a mobile device located in the real geographical position because students have to observe or interact with the environment. They need to have physical context-awareness in order to answer the question. The students have to follow a route, for this reason orchestrating the provision of questions and feedback is very important for guiding the students during the activity. The feedback has to help the students to reflect about the answers, but also has to indicate the itinerary that they have to follow in order to find the next questions. An assessment in (real) situ activity can be only performed using a mobile device; whereas an assessment in (virtual) situ activity is based on computer web access. 
Normally in a test activity teachers create the test and after its completion, they see the results obtained by the students. In the case of assessment in situ activities, a higher involvement of the teacher is required. They can know in real time where their students are and the results obtained by each student during the route and adapt the feedbacks/hints considering the students' locations.

In this paper we focus our attention on the assessment in (real) situ activities. Specifically, we analyse the use of geolocation information to make possible the automatic visualization of assessment resources when students are correctly located in the desired emplacements.

\section{The system: QuesTInSitu}

QuesTInSitu is a web-based system designed and built to support assessment in situ activities based on tests. Using its Graphical User Interface (GUI) an author can create two types of assessment in situ activities: assessment in real \& virtual situ. Currently the web map application used to play the questions is Google Maps, but it could be extended to other web map tools. In this section we describe the main functionalities of QuesTInSitu for the authors (teachers) and for the students, and the components which conforms the architecture of the system. QuesTInSitu has a mobile web version that is also explained in the next sections, it allows students to follow the assessment in situ activities, answering the questions, receiving feedback and score in situ and personalizing the questions with their own pictures and comments using a smartphone.

\subsection{QuesTInSitu functionalities}

Teachers can use QuesTInSitu to create three types of QTI v 2.1 questions: Multiple Choice, Multiple Response and Yes/No (see Figure 1). A user-friendly editor shows the necessary steps that have to be fulfilled in order to create the geolocated question. Teachers have to introduce the text of the question, the response/s and select the correct answer and feedback. After that, they have to geolocate the question in a Google Maps window, searching the position desired and clicking over the location so a coordinate is associated to the question. As a result, the question is created and positioned in the map.

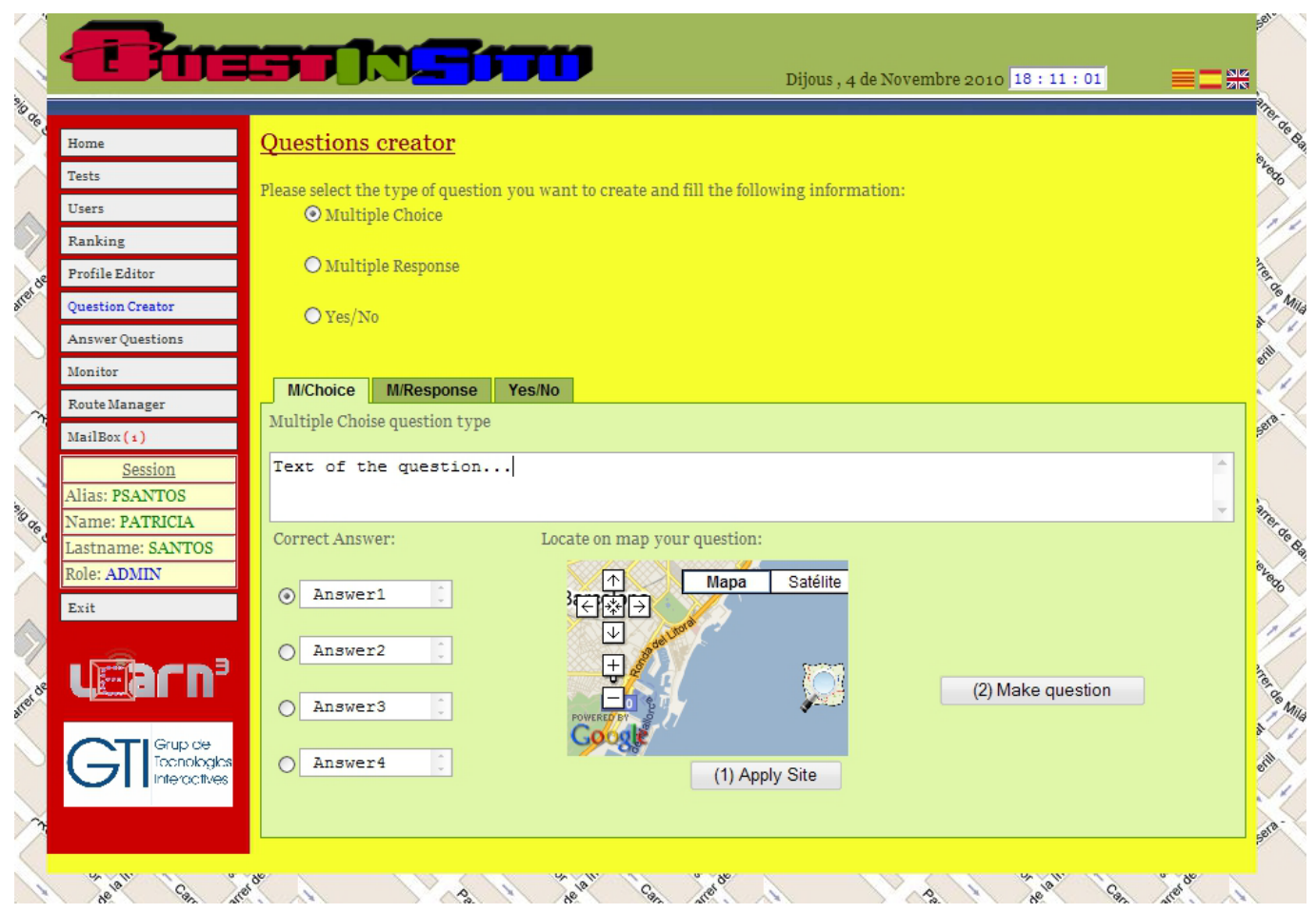

Figure 1. QuesTInSitu QTI geolocated question creator

When teachers have created a set of questions, they can create a test (or which is the same a route in QuesTInSitu) using the Route creator section. First they have to introduce the name of the route, and then that they can: (1) search and select the question that they want from a web map view (where all the questions that are located in the system are shown); or (2) select the question from a combo box that 
contains all the names of the questions saved in the system. Also authors can manage the routes created, adding or deleting questions.

In order to verify that the questions of the route are well designed, authors can check the questions directly from the QuesTInSitu player application. To do this they have to go to the Answer Questions section and select the route (test) they want to answer. A web map appears with different markers (one per question), when a marker is selected a speech bubble appears with the text of the question (see Figure 2).Once the question is answered, it is automatically corrected. When the answer is correct the bubble (and the marker) appears in green, when it is incorrect it appears in red. Before answering a question the marker is white with a red point in the middle.

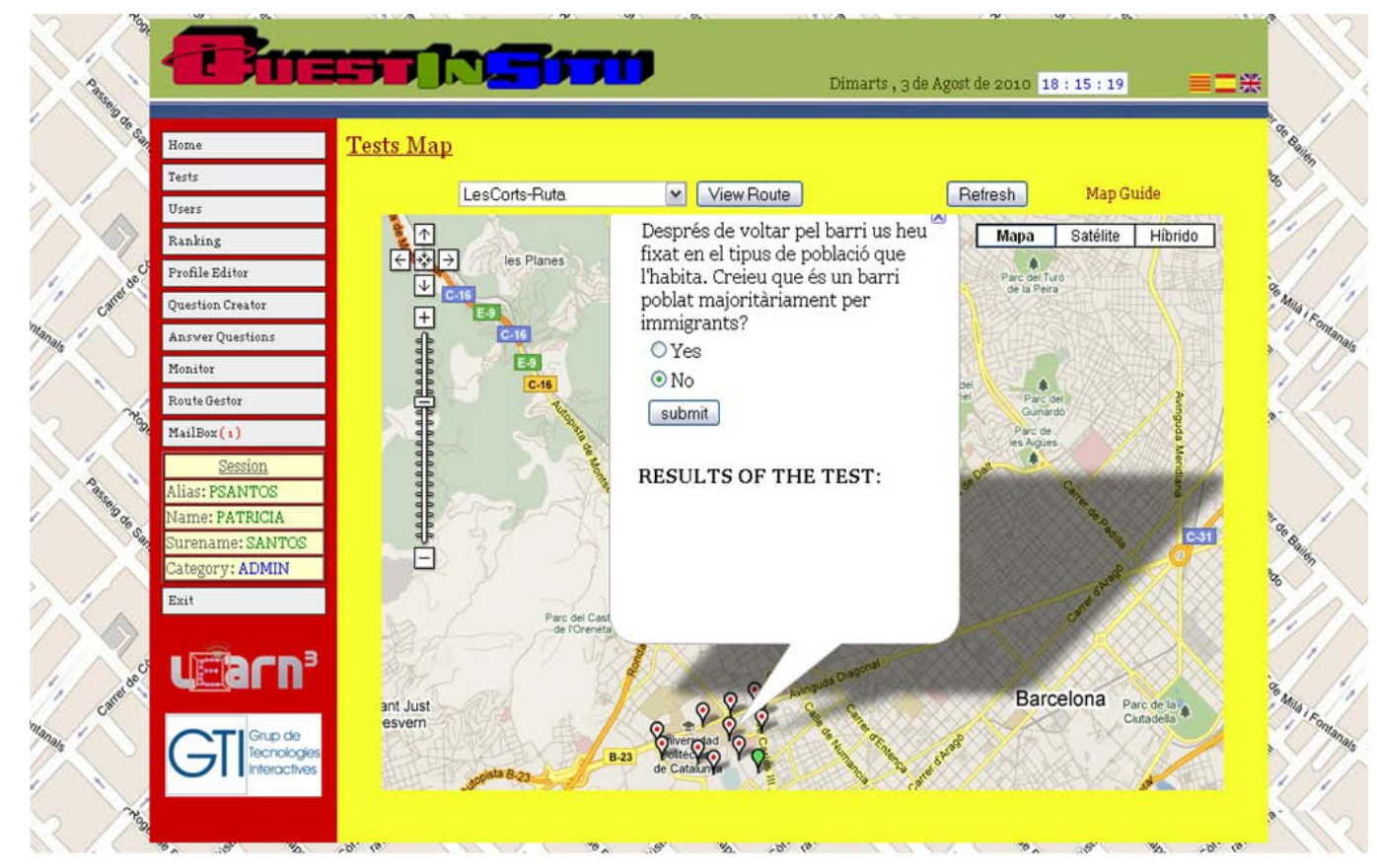

Figure 2. QuesTInSitu selecting a route and answering a question

In order to use QuesTInSitu in real situ, students have to follow the route equipped with smartphones. In the case that the route has GPS signal infrastructure, when students are positioned in a radius that contains a geolocated question, a question appears automatically in the screen of the phone. On the contrary, if the zone does not receive well the GPS signal, the questions have to be selected manually by the students. See more details about this process in The system architecture subsection.

Teachers can monitor the activity in real time using the Monitor feature of QuesTInSitu (see Figure 3). After selecting the route that they want to monitor, a set of markers (corresponding to the questions of this route) appears. The markers in yellow are questions that have been answered by the students that are doing the route. Selecting one of these yellow markers teachers know the punctuation achieved by a student in the question. With this information they can know approximately where the students are and their progress in the activity. 


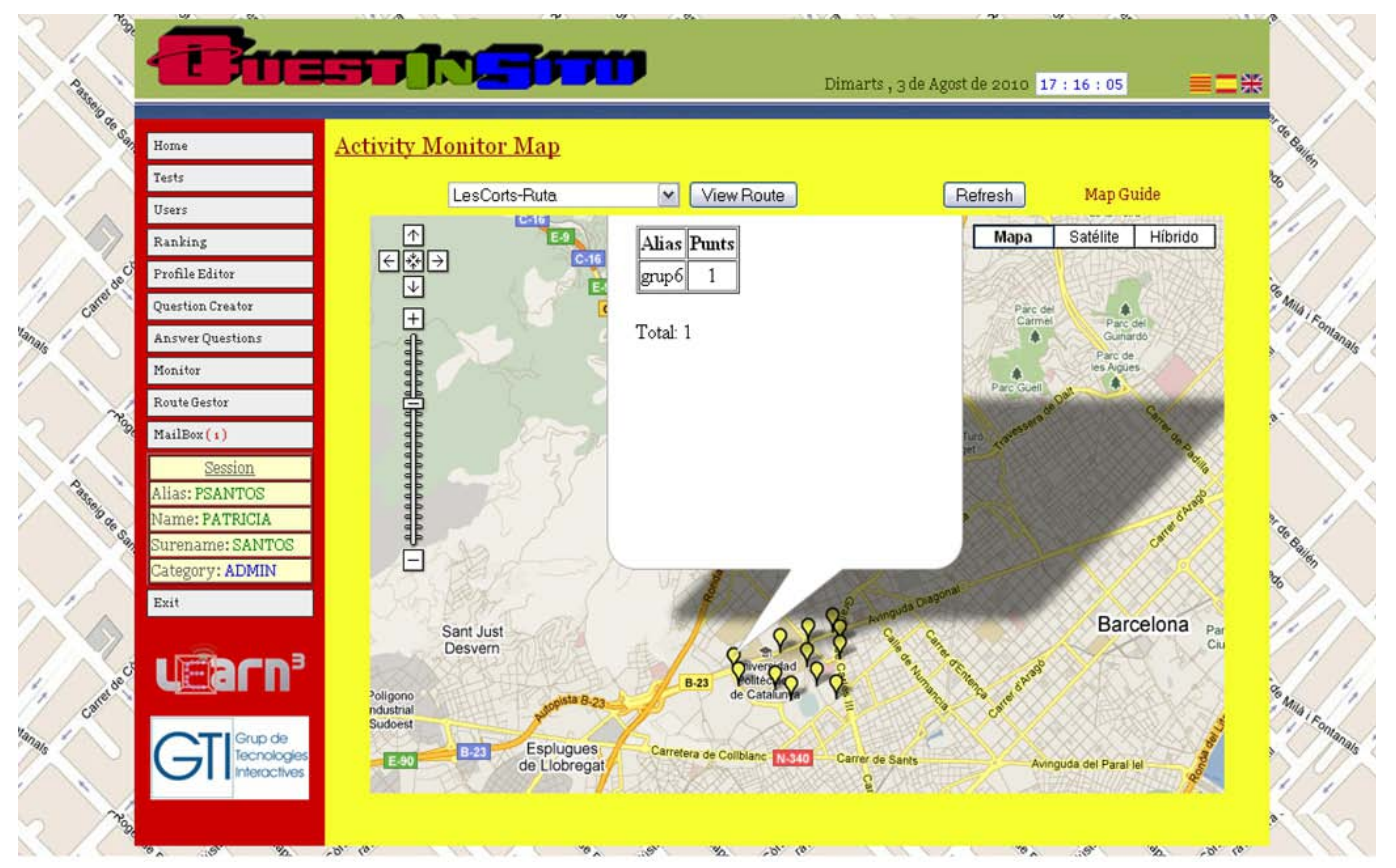

Figure 3. Monitoring group performance during the route

Once the students answer a question, the system corrects it and shows the score and the feedback associated to this question (in green when is correct and in red when is incorrect) (see Figure 4 (a)). The feedback is a message, pre-designed by a teacher, that shows which was the correct answer and also explains the next steps that the students have to follow.

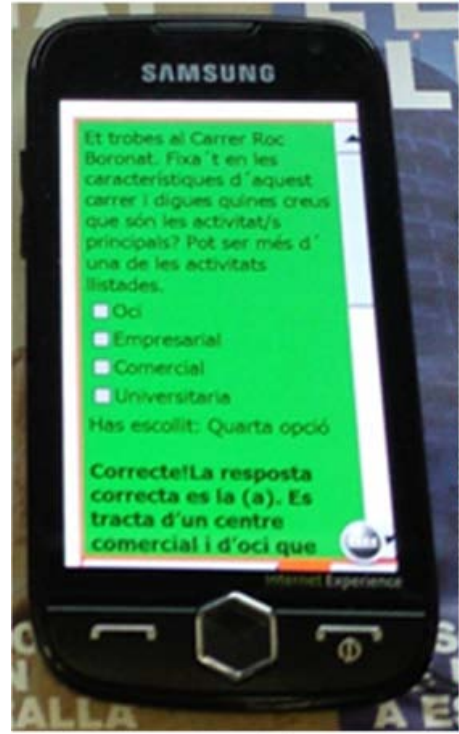

(a)

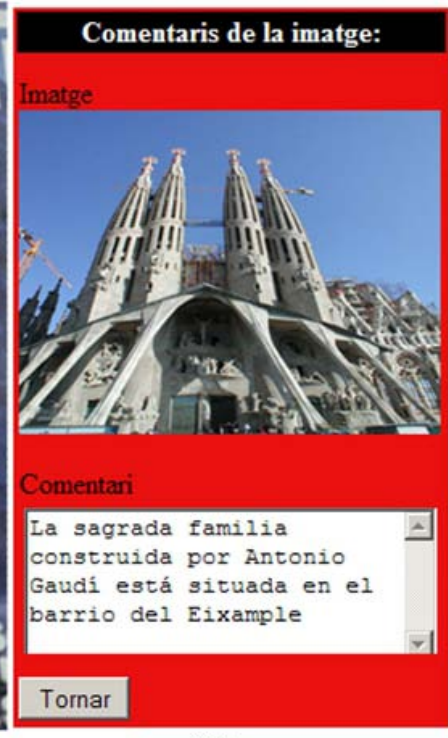

(b)

Figure 4. QuesTInSitu mobile web interface (a) Example of an answered QTI question visualized through a mobile phone (b) Example of image and comment added by a student associated to a question

The information of the answered questions is saved in the system. The students can review it whenever they want although the activity is finished. They only have to access to the QuesTInSitu system with their username and password and they can see their history of questions and responses. Moreover, the students can enrich and personalize the questions with comments and images (taken during the route with the smartphone or a camera, or downloaded from Internet), see Figure 4 (b). These data are shared with the other students that also have answered the same question. 


\subsection{The system architecture}

The architecture (see Figure 5) comprises four different components:

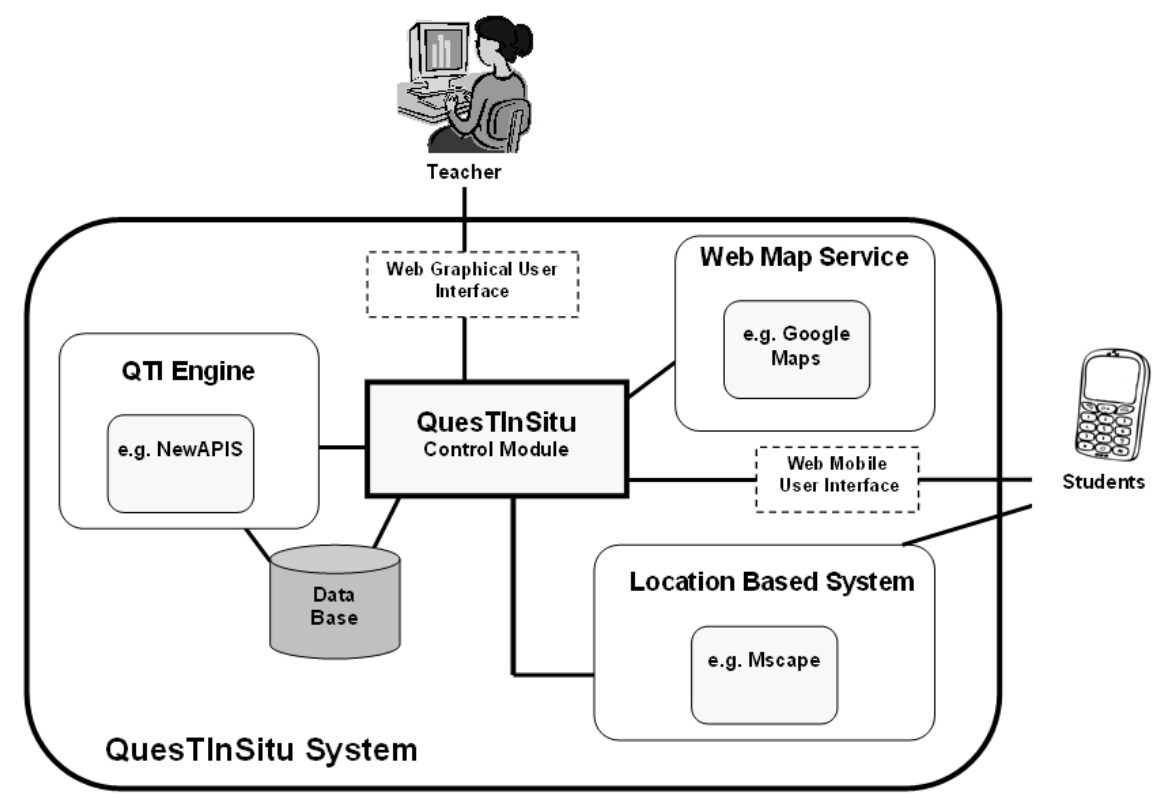

Figure 5. System Architecture

(1) QuesTInSitu is a Java Server Pages (JSP) application used to design, play and implement assessment in situ activities. Its control module has been developed to make possible the management of the following applications: QTI Engine, a Web Map Service and a Location Based System. (2) NewAPIS is the engine which interprets de QTI questions compliant with QTI v2.1). The newAPIS engine (Blat, Navarrete, Mognieh, \& Batlle, 2007) is an extension of a previous project called APIS (Assessment Provision through Interoperable Segments) (APIS, 2004) which was compliant with QTI v.2.0. NewAPIS has been extended and now is compliant with QTI v2.1. This engine in characterized for being capable of dealing with test and complex response processing. Both, QuesTInSitu and NewAPIS share the same data base storing all the information related to the questions, the test-routes, the users and the maps.

QuesTInSitu is connected with the third component (3) Google Maps. This server provides the necessary web maps to the system in order to associate questions with real coordinates and visualize them in a web map. Google Maps can be substituted in the future by another web map application (e.g. Yahoo!Maps or OpenStreetMap). Finally the fourth component (4) is a Location Based System. The version of QuesTInSitu used in the experiments of this paper did not have implemented its own module for locating the position of a mobile using the information of the GPS. This feature is necessary to know the position of a user and then throw an event with the associated question. As a proof of concept, QuesTInSitu used the Mscape system (previously described in Section 2.2) to manage the geolocated data. The maps created with QuesTInSitu and Mscape share the coordinates marked in QuesTInSitu. Mscape was installed in the mobiles, when a user was doing the route with the smartphone and Mscape received the position from the GPS. When a pre-determined position was found, a link appears in the mobile and the user could connect with QuesTInSitu in order to answer the questions.

QuesTInSitu allows students to follow the test-route while they are walking. They have to use a smartphone with Internet connection, GPS and camera. Meanwhile, teachers can monitor the student's progress on the activity via the QuesTInSitu's web Graphical User Interface (GUI) application.

It is important to remark that the architecture reflects that the most important aspect of this system is the combination of an assessment engine with a Web Map Service and a Location based System, so as to exploit their features for creating assessment in situ activities. 


\section{Evaluation of QuesTInSitu}

The objective of this evaluation is to find results that help us to validate the following statement: "Enriched assessment activities that facilitate the students' learning in situ can be achieved with the QuesTInSitu system.”

This section presents three scenarios evidencing the value of the QuesTInSitu system for creating assessment in situ activities (see Figure 6). In the experiment, a real educational activity for learning town-planning concepts about the city of Barcelona has been evaluated and put into practice with 34 students (boys and girls with an average of 17 years old) and two teachers. The QuesTInSitu system was used to enhance an activity performed the previous years. The technological solution facilitates the automatic provision of questions when students are located in the correct positions. Besides, teachers can monitor the students' responses and the provision of feedback and score in real time. In the experiment we analyse how the use of the GPS (versus not using it) affects the performance of the activity and students' behaviour. The evaluation of the experience is performed with the aim of analysing the learning benefits, for students and teachers, of the assessment in situ activity created with QuesTInSitu.

Next, with the objective of illustrating that the QuesTInSitu's test-routes are useful to enrich existing assessment practices two examples of scenarios are also included in the paper. The first example, example A, helps us to show that QuesTInSitu can be used to enhance a ubiquitous approach proposed by others authors. Huang et al. (2009), present a Mobile Plant Learning System that facilitates the learning of botany using PDAs and GPS in open spaces. Using QuesTInSitu the activity can be enriched by means of the inclusion of automatic assessment in situ. In the second example, example B, a hypothetic situation based on real practices, exemplifies that QuesTInSitu can be used to facilitate and support the performance of cultural itineraries with assessment purposes. The system offers the possibility of geolocating questions in the same geographical coordinates that the selected itinerary has.

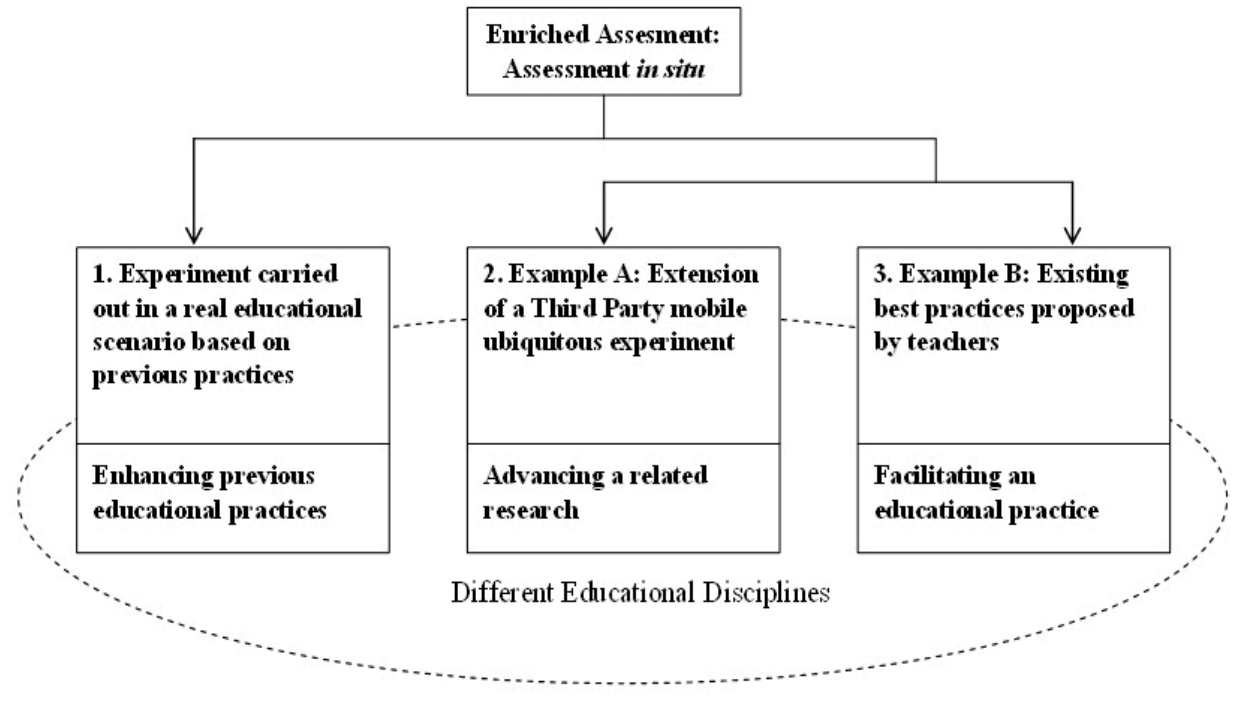

Figure 6. Graphic representation of the scenarios used in the evaluation

The first scenario, Discovering Barcelona, is the scenario which has been evaluated with real students and teachers. This scenario includes on the one hand the description of the design of the experiment and on the other hand the analysis of the experiment following the guidelines defined by the evaluation method proposed in (Martinez, Dimitriadis, Rubia, Gomez, \& de la Fuente, 2003). The research question of the experiment was focused on analysing the educational benefits of using QuesTInSitu with smartphones to perform an assessment in situ activity.

Then, example A and B are presented in the following section because these scenarios have not been evaluated following an experiment with real users. These other scenarios have been included to illustrate different uses of QuesTInSitu for different educational disciplines.

\subsection{Experiment design: Discovering Barcelona with QuesTInSitu}


A real assessment in situ activity using QuesTInSitu was implemented and realised by a group of high school students and their teachers. The activity was an adaptation of an activity carried out in previous years where teachers explored with their students a district of Barcelona (without using any technological solution). The aim was to use QuesTInSitu to enhance the activity with enriched assessment in situ.

\subsubsection{Educational Context}

The educational context was framed at the Duc de Montblanc High School in Rubí, Catalonia (Spain). The assessment activity involved a Geography and History class with 34 students, 12 boys and 22 girls aged between 17 and 19 years, and two teachers (the main teacher and an assistant). This is a mandatory subject in the second year of the humanistic high school degree. One of the topics that the students learn during the course is the Barcelona's town planning. The teachers explain the main characteristics of the different districts (architecture, society, public services, etc.). In past years they organized an activity to visit a district of Barcelona. In this activity, the students carried a dossier with questions that they had to complete during the visit or at home. In the meantime the teachers accompanied the students and explained the main characteristics of the area. After the visit, the students had to prepare a work of the district using the questions that they completed and their notes. The main teacher had an experience of 30 years teaching Geography and History, and the assistant of 4 years. The main teacher was in charge of teaching and assessing all the material contained in the educational curriculum of the subject. The assistant helped the main teacher in particular occasions such as the realisation of an activity outside the classroom. Neither of them had experience using smartphones nor mobile devices with learning/assessment purposes. But they thought the inclusion of these technologies would improve the students' motivation to learn Geography and History.

Two researchers and the teachers had meetings and exchanged information with the objective of identifying the main problems of previous activities. The re-design of the activity was discussed and agreed with the teachers following a participatory design process (Schuler \& Namioka, 1993). The problems detected were: (1) Students only visited a unique district with their teacher. Teachers had to accompany the students to verify that students were following the correct itinerary and exploring the environment during the route. It was a hard task to monitor the work done by the students during the route. (2) The students answered questions during the visit but they did not know the correct answer of the questions until the teachers corrected the tests (because they did not have time during the route to attend every student). The students lost the opportunity of reflecting in situ about the answers to the questions.

The discussions led to re-design QuesTInSitu and to propose an experiment to analyse the new assessment in situ activity for discovering Barcelona. The objective was to enhance the old activity using a technological solution. The QuesTInSitu system had to allow teachers to distribute their students around different districts. The teachers should know the location of their students in real time. The solution should also help students to reflect in situ the answers delivered and to guide them during the route. These aspects were introduced as functionalities in QuesTInSitu in order to create a formative assessment in situ activity.

When the system was ready to be used, the teachers decided to make 6 groups of students. Each one would visit a different district of Barcelona (Les Corts, Eixample, Sarrià. Gràcia, Sant Martí and Ciutat Vella). QuesTInSitu was used to create the questions of a total of six routes. Before doing the real experience, the researches performed some trials where they detected that the integrated GPS of the mobiles did not work in three of the six districts (Gràcia, Sarrià and Ciutat Vella) due to the architectural characteristics of some streets. And in one district (Sant Martí) the GPS only worked in the half of the route created. The solution applied was that in the three cases that the GPS did not work, the students would have to select manually the questions (from an HTML page that would be included in the mobile) when they were in front of a question position. To know when a question has to be selected, the students would carry a paper map that would indicate the position of the questions. For the two districts (Les Corts and Eixample) where the GPS worked perfectly and for the district that the GPS worked in the half of the route (Sant Martí) the researchers installed Mscape in the mobiles. They adapted the routes and events designed with QuesTInSitu and created the maps in Mscape which were saved in the mobiles. This task was done by the researchers because the teachers did not have the enough technical knowledge to use Mscape and install it in the mobiles. When the groups with GPS would be following the route the events would appear automatically in the mobile and the students could read and answer the question. The groups of Les Corts and Eixample districts would carry with themselves a paper map where the position of the questions would not be indicated. In the case of the Sant Martí district, the group would carry a paper-map where only the positions of those questions where the GPS did not work would be indicated. 
The groups that used the GPS and the groups that not used it were compared, so as to understand the differences between the groups during the performance of the activity.

More details about the different tasks of the activity are explained in section 4.1.4.

\subsubsection{Research question and objectives}

The experiment was realised with the objective of answering the following main research question: Can the QuesTInSitu system support an assessment in situ activity by facilitating learning benefits that are difficult to achieve without technology?

In order to satisfactorily answer this question the results obtained after the evaluation of the Discovering Barcelona experiment must demonstrate that QuesTInSitu accomplishes the following features:

\section{QuesTInSitu must be able to:}

- Give support in situ for students during a ubiquitous assessment activity. Students have to be able of answering the questions in situ, receiving the feedback and the score immediately afterwards. The provision of the feedback in situ has to help them to reflect about the question in the adequate spatial location. Besides the feedback also has to help them to find the correct way of the route.

- Allow students to share their knowledge. The students have to be able of personalizing the questions with pictures of the place and their own notations. This information has to be shared with other partners that also have answered the same question.

- $\quad$ On the other hand, it has to facilitate teachers the task of creating assessment in situ activities. Teachers have to be able of creating the questions and test-routes that conforms the activity using the functionalities provided by the QuesTInSitu's GUI.

- And also, during the realization of the activity, teachers have to monitor in real time the progress of the students. The information provided to the teachers has to be useful for controlling the effectiveness of the activity. Teachers need to know where their students are located during the activity in order to detect that students are following the route correctly. Also they have to know whether students are answering correctly the questions, in order to detect misunderstandings.

- The way of structuring the activity with smartphones and QuesTInSitu during the ubiquitous assessment activity has to encourage the students to explore the environment. The technology used has to be useful to motivate the students to answer geolocated questions. Pintrich (2003) claims that from a motivational perspective the role of students' motivation in learning contexts helps to understand whether students learn and thrive. An important task during the evaluation is to detect the most motivational elements of the activity.

- Moreover, the activity has to facilitate the student learning about town-planning concepts. The results of the evaluation have to evidence that the technological solution helps the students in their tasks during the activity and have a positive effect in their acquisition of learning.

\subsubsection{Methodology and Data Gathering Techniques}

The mixed method proposed by Martinez et al. (2003) has been selected for doing the evaluation of the experiment due the characteristics of the educational context. This is the most suitable method because its mixed nature provides guidelines that facilitate the understanding of the experiment. On the one hand, the method indicates that qualitative data such as the participants' perspective can be collected using data sources such as: observations, interviews and questionnaires (with open questions). On the other hand, the guidelines of the method also indicate that it is necessary to collect quantitative data such as: the occurrence of actions during the activity, the data saved in QuesTInSitu or the answers to closed questions in questionnaires. Quantitative data is used to detect tendencies, which are confirmed or rejected through the analysis of the qualitative data. The different data gathering techniques used for the evaluation are described in Table 1.

After using the mixed evaluation method to collect data, two researchers followed a member checking approach in order to analyze the information achieved. The data collected from the students and teachers questionnaires, direct observations during the experience (with observers' notations and video recordings), and interviews, were first analyzed and next triangulated by each researcher separately and 
finally compared in order to obtain trustworthy results. Member checking is identified by Lincoln \& Guba (1985) as the best method for increasing the credibility of the analysis of qualitative information.

Table 1. Data collection techniques

\subsubsection{Experiment procedure and measurement techniques}

In April 2010, the formative assessment activity called "Discovering Barcelona!” was performed. It was divided into four different phases: (1) Assigning Districts, (2) Discovering the District, (3) Reflect about your district and (4) Test your colleagues. Phase 2 was the assessment in situ activity, this paper focuses the attention on this phase but we also explain the others because it helps to understand the whole activity. The phases are explained in what follows:

(1) Assigning Districts (Diagnostic Assessment): before going to visit the districts, the teachers explained the activity to the students. The researches sent an e-mail to all the students with a prequestionnaire [Pre-Quest]. The questionnaire was used as a method of diagnostic assessment, to know which district the students preferred to visit, but it also contained questions about the six districts. The preferences, the answers to the questions and the teachers' criterion were used to assign districts and form balanced groups. On the one hand, when the majority of the students in the same group answered incorrectly a question of a specific district then the district with more fails was assigned to the group. On the other hand, when the majority of the students of a group answered correctly the questions of the six districts then the district assigned was the most voted by the members of this group.

(2) Discovering Barcelona (Assessment in situ): in this phase the students visited the six selected districts of Barcelona. The activity consisted in assessing the transversal and specific knowledge and skills of the students in situ, using the routes designed by the teachers. Each route had QTI questions (with an average of 12 questions per route). The final score obtained after doing the test-route counting for the students' final mark. Each group of students carried a mobile with GPS, Internet and camera. The mobiles phones were the Samsung Omnia I and II models. As we mentioned above, in some districts the GPS did not work correctly, for this reason the groups were organized as follows: Eixample and Les Corts (used GPS), Sant Martí (used in some positions of the route the GPS) and finally, Ciutat Vella, Gràcia and Sarrià (did not use the GPS). All the students had to answer the QTI questions when they were located inside of a question-area. The consequences of using (or not using the GPS) were compared and evaluated during the experiment in order to understand the benefits/limitations of using GPS to control the visualization of questions.

Due the novelty of the experiment the students visited the district assigned in groups. Every group was accompanied by a monitor who assigned roles for giving a responsibility and involve each student in the assessment activity: the person who carried the mobile and selected the questions was the "Mobile Phone Manager", the "Photographer" who took pictures of the district, the person who took notes of the correct and incorrect questions was the "Question Helper", the "Observer" who annotated the characteristics of the district, and finally the person who carried the map of the zone who was the "Guide". When groups had more than five students some of them played the same role.

The groups had 2 hours for completing the route. During the route they had to answer a set of questions which were related with the characteristics of specific important areas of the itinerary. When the group was in an area with a question, if they used the GPS with Mscape an event appeared in the screen of the phone alerting them that they were in a question-area. Then the students press a link that connects with the associated question in QuesTInSitu. In the cases that the groups did not use the GPS the Guide student had to alert the Mobile Phone Manager that they were in an area with an associated question. In both cases the Mobile Phone Manager selected and read the question aloud. The groups discussed the possible answers looking at the environment or asking to the citizens who were in the zone. Finally the responsible of the mobile had to select the answer (or answers) that the group decided as correct. After it, QuesTInSitu computed the answer and showed the result (correct or incorrect) and the feedback. The Question helper annotated the result. They read the feedback that indicated which streets they had to follow in order to arrive to the next question-area. During the route the Photographer took photographs of the streets, buildings and other elements. The Observer wrote the main characteristics of the district in relation with the questions. Once they finished the route, the monitor explained them that they could check the questions and their answers in the QuesTInSitu system. They could also use the system (using the computer or a smartphone) to upload the pictures and comments and associate them to each specific question. At the end of the route the punctuations achieved by all the groups were stored in QuesTInSitu [Automatic-Data]. During the route the monitors took observations [O-st-route]. Finally the students 
answered a questionnaire [Q-st-route] distributed by the monitor to collect quantitative and qualitative data from the students to evaluate the experience.

During this phase, the teachers were located in a room monitoring the activity done by the six groups using the QuesTInSitu application with a computer. They could select one of the six possible routes and know in real time where the students were located, which questions they have answered and punctuation obtained. When the activity was over the teachers answered a questionnaire [Q-t-route \& O-t-route].

(3) Reflecting about your district (Demonstrate the knowledge and skills achieved during the assessment): Two weeks after the visit to the districts, the students did a presentation talking about the district visited. For doing the presentation the students used: the QuesTInSitu application (to review the questions and answers), adding the pictures and comments that they took during the visit and also they used information extracted from Internet. The objective of this activity was to share with their class-mates the knowledge achieved about their district. All the presentations were uploaded to a Moodle course designed for this occasion. During the presentations the researchers took observations and made a video [O-st-final]. When the presentations finished, the students answered a final questionnaire [Q-st-final] and the main teacher of the subject answered to some questions of an interview and to a final questionnaire [Teacher-comments \& O-t-final \& Q-t-final].

(4) Testing your colleagues (Self-assessment): Since the end of the activity coincided with the beginning of the final examination period of the course, the last phase was optional. In this phase students were invited to participate in the QuesTInSitu application to check their position in the final ranking, to create new questions, or to answer the questions that their class-mates answered in their routes.

See description of the data sources at Table 1.

\subsection{Results and analysis of the Discovering Barcelona experiment}

The three main results (and partial results) after the evaluation of the Discovering Barcelona experiment are summarized in Tables 2, 3 and 4. These results are used to answer the research question formulated in section 4.1.2.

\subsubsection{Result I: QuesTInSitu supports real assessment in situ activities}

Part of the data collected during the experiment (see Table 2) allow us to present the first result that states that QuesTInSitu gives a valuable support for teachers and students in the performance of a real assessment in situ activity in open real world environments.

Table 2. Result I: QuesTInSitu for supporting assessment in situ. Summary of partial results and supporting data

More specifically, as the partial results of Table 2 show, the two teachers indicated that QuesTInSitu is very useful in education and helps students to create and follow an assessment in situ activity in an open real world environment. The teachers valued several factors of the system as useful but one of the factors that the teachers found more helpful was the monitoring in real time service. Teachers punctuated this functionality with the highest mark of 5/5 points [Q-t-route]. The teachers indicated that using QuesTInSitu the students have the possibility of being more autonomous. One of the teachers' comments that support these statements was: "(Teacher 1) It allows you to design activities (test, routes ...) and see from a fixed location the progress of the activity (where the students are and what questions they have done, what have answered correctly or not...)" [Q-t-route]. Also the teachers used the following adjectives to define QuesTInSitu: “(Teacher 1 and 2) ... practical, rational, specific, intelligible and functional" [TeacherComments]. Even the teachers do not usually use computers they understood quickly all the possibilities that the tool has.

From the point of view of the students, the majority of them (97\%) indicated that QuesTInSitu was very practical and easy to use and learn. This result is also supported by the punctuations that students rated regarding the tool, where the majority of the students punctuated it with the highest marks: $65,5 \%$ of the students with $4 / 5$ points and the $13 \%$ with $5 / 5$ points. $20 \%$ of the students punctuated the tool with $3 / 5$ points [Q-st-final]. These data supports the good acceptance of QuesTInSitu. A common comment was that the process that the students had to follow for answering the questions was very easy to understand. Only with a short explanation at the beginning of the activity the students used QuesTInSitu without learnability problems. Some students' comments also support these statements: "(Sant Martí - student 4) It (the system) indicates the steps to follow in a very clear way"; “(Sarrià - student 1) It's been easy because everything is guided and structured in an easy way" [Q-st-route]. The partial results obtained 
after analysing the data collected in the experiment, indicated that the students understood the objectives of the tool and for this reason they valued positively the QuesTInSitu's functionalities that were designed to help them during the activity. Students were very enthusiastic when they received the feedback and the score of the questions (especially when the answer selected was correct). The $97 \%$ of the students indicated that the feedback in situ helps them to know how to continue in the route of the district assigned and the score shows their progress on it. The $100 \%$ of the students said that they liked to receive the feedback and score in real time [Q-st-route]. Students did comments such as: "(Eixample student 2) In real time it (QuesTInSitu) gives you the answer and it guides you on the route" and "(Sarrià - student 1) ... answering the questions was easy because we were in front of the location related with the question" [Q-st-route]. These comments indicate that the provision of feedback in situ and in real time, help the students to understand better the assessment activity. Then we can state that the provision of feedback in situ is an incentive for the students to follow with the activity.

Other actions that demonstrate that they understood quickly the functionalities of the system were that some students consulted the information saved in QuesTInSitu about the feedback obtained in past questions to remember their feedback messages and scores. Besides the students were very interested in knowing their position (final score obtained in the route) indicated in the ranking. The teachers commented that for the students the "numbers" (marks) are very important. Some reactions observed during the visualization of the ranking in classroom after doing the activity of discovering the district were: "(Observer 1) Students are excited when the researcher is showing the positions on the ranking. The group who is in the first position joyed: We won!; and some class-mates made jokes answering: For sure you cheated!" [O-st-final].

Both, teachers and students suggested some improvements for the QuesTInSitu's functionalities. These comments demonstrate the level of expertise that the participants acquired in a short period of time. On the one hand, teachers suggested to (1) make a section with the final mark of each group in a specific test. At the end of the experience the researchers considered this suggestion and they added a final report of each group in the ranking section. Teachers also wanted to (2) have more types of questions (e.g. open questions, questions with images/audio/video); and also they wanted to (3) extend the monitoring system by adding an audiovisual and communication system to talk with the students on runtime. The teachers said: "(Teacher 1) It would be very interesting if it's possible to follow the students visually with a system (like a video)" and "(Teacher 2) I would have wanted and audio-visual system" [Qt-route]. On the other hand, students also indicated some problems using the functionality of personalizing their assessment resources. The $52 \%$ of students uploaded assessment resources (images or comments) to QuesTInSitu. The $26 \%$ of the students had problems uploading images and the $8 \%$ editing a comment of a question. The comments were considered during the activity and were fixed by the researchers. Students also suggested adding videos as assessment resources of a question.

\subsubsection{Result II: QuesTInSitu facilitates learning achievement}

The second result, claims that the combination of elements that conforms the QuesTInSitu system (an Assessment Engine, a Web Map Service, a Location Based System and the use of computers and smartphones with GPS and Internet) allows performing an enriched assessment in situ activity that facilitates the practice of transversal and specific knowledge and skills (see Table 3).

Table 3. Result II: QuesTInSitu for mediating assessment in situ activities with educational benefits. Summary of partial results and supporting data

As we have explained previously, in previous years the teachers made a similar activity without using technology for mediating the discovering of the district. They used paper-based exercises to assess the students' knowledge, exercises that they had to answer in classroom or at home. After doing the activity using the QuesTInSitu system solution, the teachers indicated that the use of this technology helps to structure the activity in order that the students achieve more skills: orientation and explorative skills, and moreover students are more autonomous because they have to explore the district by themselves. An observation noted during one of the interviews with the teachers: "(Observer 1) The teachers think that students have learnt urban information in this activity and that, in an exam they will just 'vomit' (Catalan expression meaning that students answer without thinking) all what they know. However, going to the particular locations and think about the place, make them reflect about what they learn" [TeacherComments]. An opinion wrote by one of the teachers concerning this issue in one of the questionnaires was: "(Teacher 2) Students could learn about more districts working with mobile devices enable achieving other learning objectives: orientation and explorative skills and a more personal observation (of the environment)" [Q-t-route]. 
From the point of view of the students as it can be appreciated in Table 3 the $94 \%$ of the students indicated that they preferred answering the questions using mobile devices instead of filling in a dossier or doing an exam in class. Only the $6 \%$ of the students indicated that they prefer to answer the questions in a dossier or doing an exam. When the questionnaire asked about which type of method (1) the mobiles for answering in situ or (2) the dossier with questions and an exam, is better for learning. The $82 \%$ of the students selected the first option and the $12 \%$ the second (the $6 \%$ of students did not answer this question [Q-st-final]).One student commented: “(Sant Martí - student 5) ... looking the places you answer better the question. When you answer a dossier with questions after doing an activity (outside the classroom) you do not remember all". On the other hand a student who selected the option (2) answering the questions in a dossier, said: "(Eixample - student 1) with this option you have the teacher for helping you" [Q-st-route].

The students commented the advantages of structuring the assessment in situ activity for discovering the district with QuesTInSitu and mobile phones: (1) The phone is easier of carrying than a dossier with questions. (2) Answering the questions in front of the associated space and receive the feedback help them to understand better the question. (3) They were more concentrated in the activity, because with the questions appearance they could notice more details and learn more. (4) The distribution of roles helps them to participate actively in the activity. (5) The activity was more dynamic in comparison to other activities (e.g. visiting a museum). (6) In addition the students highlighted that after the experience they have learnt how to use a LBS device. The comments concerning the benefits of the assessment in situ activity made by students were: "(Eixample - student 2) I liked to have the feedback with the answer in real time, because then you can know where you are wrong and in addition it (the feedback) guides you on the route"; "(Les Corts - student 4) I liked to know the score in situ because then you know if you observe enough the environment" ; "(Gràcia -student 5) The activity was more dynamic, you had to take part and to know what to do"; and "(Eixample - student 5) ...it is an innovative method that allows the student to feel useful in the activity" [Q-st-route].

Considering the comments and the observations taken during the experience there are some preliminary evidences that show that the students achieved new skills and knowledge doing the exploration of the district and listening to the presentations of their classmates in comparison to previous experiences (where the students did not use technology). The summary of the analysis of these observations is presented as follows: (1) The students put in practice their social and communicative skills talking with the people of the district to obtain hints for answering the questions; (2) The observations did during the activity verify that the members of the same group put in practice teamwork skills because they discussed and negotiated before answering the questions. They were interested in knowing the opinions of all the group and they wanted to discuss in order to find the better answer (critical thinking skills). (3) When a question appears in the smartphone the students observed the environment, taking notes and pictures. (4) After receiving the feedback, they reflected and confirmed the correct answer looking the environment. (5) The role distribution was important for doing the activity. This distribution affected in the students' positive interdependence and helps them on focusing on the task. (6) The students found that using a mobile phone, a map and the GPS they learnt how to orientate and locate themselves in the district. (7) The $97 \%$ of the students indicated that after the experience they have learnt new town-planning concepts about the districts. And finally, (8) the feedback in situ helps them to understand better the question and to learn more about the content related. As an example part of these statements can be appreciated in the following observations noted during the activity: "(Observer 5) The question 6 suddenly appears and we are in front of the building related with the question. The students read the question and look the name of the building, they said: Look this is the answer, it is the Scientific Park!. Then they answered correctly the question"; "(Observer 6) Students are making photographs constantly and they talk about the content of the question" [O-st-route]. Also the statements can be confirmed with the remarks made by the students in the questionnaires: "(Gràcia student 4) Using the mobile phone you have to pay attention constantly"; "(Les Corts - student 6) ...all the members gave our opinion in order to obtain a conclusion"; "(Ciutat Vella - student 1) I have learn how to situate myself in the district” [O-st-route, O-st-final, Q-st-route \& Q-st-final].

After doing the whole activity the students valued in a likert scale their perception of learning: the $18 \%$ selected $3 / 5$ points; the $68 \%$ valued with $4 / 5$ points; and the $6 \%$ with $5 / 5$ points. The $8 \%$ did not answer this question [Q-st-final]. A quantitative evidence of the learning achieved during the activity was the final mark of the test-route. The automatic marks generated by QuesTInSitu show that any of the groups failed more than 2 questions: 1 group had a mark of 8/10 points, 4 groups had a mark of 9/10 points and 1 group has 10/10 points [Marks].

The students used the information showed during the assessment in situ activity in their final presentations in the classroom. For instance when they described the streets/buildings they used concepts 
that were explained in the feedbacks. All of them used a map to locate the district visited. They combine pictures taken during the activity with pictures extracted from Internet.

Although the students valued positively the use of smartphones for doing an assessment in situ activity they also detected some technical limitations and problems. Half of the groups encountered technical problems. At least in one occasion during the route (1) the mobiles phones did not work properly or worked slowly. Also (2) the tactile screen did not detect always the interactions when they tried to answer a question. Finally (3) two of the total of three groups that used the GPS indicated that it did not work properly in all the occasions. This can be appreciated in the following student's comment: "(Sarrià-student 3) It is a good idea to use mobile applications but sometimes (the mobile) works slowly or it did not works properly" [Q-st-final].

As we have indicated previously, the use of the GPS to throw questions detecting the position of the students was a control factor due the characteristics of the streets of the six different groups. Two groups used the GPS in the whole route, three groups did not use the GPS and one group, the MIXED, used the GPS in a half of the route. The GPS was evaluated in order to understand its use and importance for doing an assessment in situ activity: (1) The GPS-groups showed a lot of curiosity and surprise knowing that the questions will appear using the GPS signal. They think that it helps them to know that they were well located. The following comment was done by a student that used the GPS: "(Les Corts student 5) It (the GPS) helped us to situate ourselves on the route because it is a good indicator for locating the streets" [Q-st-final]. (2) The No-GPS groups think that the GPS was not necessary to do the activity. But some of them indicated that the automatic appearance of questions using the GPS could help them to find quickly the correct streets where the questions were located. They commented that not using the GPS they had the feeling of interacting more with the people of the street (asking about the streets). Some students said that the GPS would help them in the task of interpreting the map. Students commented: "(Sarrià - student 3) I think that using the GPS we would had found the questions faster" and "(Gràcia - student 1) ... without the GPS we did a good work, answering the questions and following the route" [Q-st-final]. Finally (3) the MIXED group preferred the questions which appeared using the GPS because they think that when using it the activity was more dynamic, interesting, practical and faster. This idea can be confirmed with the following comments: "(Sant Martí - student 2) I think that it was more interesting when the questions were indicated mediating the GPS because the process was more dynamic and different" and "(Sant Martí - student 5) The activity was more interesting when we use the GPS because when we were advancing (on the route) in different places the GPS made that appeared questions" [Q-st-route]. The final score obtained in the routes did not differ between groups (using or not using the GPS). The partial results demonstrate that controlling the automatic questions visualization with the GPS signal is not essential for doing an assessment in situ activity. But the automation is positively appreciated by the students because it helps the students in their exploratory tasks.

\subsubsection{Result III: QuesTInSitu enacts learning attitude}

Finally the third result concludes that mediating an assessment in situ activity with QuesTInSitu and mobile technology enact the students' motivation and produces a positive impact in the teachers and students' interest and the associated learning benefits that this entails (see Table 4).

Table 4. Result III: Student and Teacher Motivation during and after the experience. Summary of partial results and supporting data

\begin{tabular}{ll}
\hline \multicolumn{1}{c}{ Result III } & \multicolumn{1}{c}{ Partial Results } \\
\hline & $\begin{array}{l}\text { All students (34/34-100\%) and the teachers indicated that would repeat the } \\
\text { experiment another year. }\end{array}$ \\
\cline { 2 - 2 } $\begin{array}{l}\text { The assessment in situ } \\
\text { activity was perceived as } \\
\text { an innovative solution } \\
\text { that had a notable }\end{array}$ & $\begin{array}{l}\text { 24/34 (71 \%) students punctuated the activity with 4/5 points and 10/34 (29 \%) } \\
\text { students with 5/5 points. }\end{array}$ \\
\cline { 2 - 2 } $\begin{array}{l}\text { positive impact in the } \\
\text { attitude/motivation of } \\
\text { the students and teachers. }\end{array}$ & $\begin{array}{l}\text { (they like to use technological devices); (2) They did the activity without the } \\
\text { teacher (3) They learnt new things about the district and how to use specific } \\
\text { technology; (4) The activity promoted their participation (in comparison with } \\
\text { other activities where the teacher was always talking and they took notes) }\end{array}$ \\
\cline { 2 - 2 } & $\begin{array}{l}\text { The teachers were very happy after the experiment and they thought that the } \\
\text { activity was a success. }\end{array}$ \\
\hline
\end{tabular}

In this sense, Table 4 shows that the $100 \%$ of the students and the two teachers commented that they would repeat the experiment in the same conditions. Specifically students rated their degree of 
satisfaction doing the whole experiment in the final questionnaire with very high punctuations: the $71 \%$ with $4 / 5$ points and the $29 \%$ with $5 / 5$ points [Q-st-final \& Q-t-final].

On the one hand a summary of the students' comments that confirms that the activity was more enjoyable and helpful for learning town-planning than other explorative activities where students /teachers did not use technology. The benefits identified by the students are: (1) The activity was very innovative and funny because the use of technological solutions; (2) They enjoyed the activity because they did it in groups and without the physical presence of a teacher; (3) They learnt new things about the district and also how to use specific technology (like a smartphone with GPS); and finally (4) the structure of the assessment in situ activity promoted the students' participation (in comparison with other activities where teachers talk and students take notes). Comments concerning these issues were: "(Les Corts - student 6) Yes, I would repeat this activity because it has been very amazing and I think that you learn more doing it"; "(Sarrià - student 4) The mobile phone is more practical than a portfolio (where the sheets are always wrinkled and you lose them...). The mobile also incites you to participate more ..." [Q-st-final].

When the whole experiment finished the teachers were satisfied because the technological solution supported successfully the exploration of the districts and the assessment of the students' knowledge and skills. Before this experiment they had never imagined the possibility of using an application mediated with mobiles for doing an assessment in situ activity. After doing the activity they would repeat it [TeacherComments].

\section{Other assessment in situ scenarios using QuesTInSitu}

Examples A and B have been included to illustrate that QuesTInSitu can be applied in a variety of educational disciplines. These scenarios have not been evaluated. However, each scenario is explained considering a research question.

\subsection{Example A: Using QuesTInSitu for including assessment in situ in a Mobile Plant Learning activity}

This scenario is presented with the objective of explaining the next research question: Can the QuesTInSitu system be used to enhance an existing ubiquitous educational practice including assessment purposes?

In order to answer this question, this scenario shows how QuesTInSitu could be combined with a mobile-ubiquitous learning system presented by others. Huang Y. et al (2009) have created a Mobile Plant Learning System (MPLS) that facilitates elementary-level science students learning about plants. Their objective was to create a system that enables the students to learn about plants and to put in practice their knowledge doing context-awareness activities outside the classroom. For this reason the MPLS was designed to be used in a PDA with GPS. The system has an environment sensing method: it can detect the student's location (through the GPS information). Then, it identifies the plants' information from its own data base that has a relation with the position of the students. When the system finds information, it displays an electronic map (e-map) in the PDA's screen, allowing the students to know the location of the plants in the area. Once the students are in front of a plant, they can find information about it in the MPLS or add pictures or comments to the system in order to share information. The authors evaluated the system with 32 students. After doing the activity the authors used a paper test to assess the students' knowledge. They conclude the experiment with positive results that reveal that this system can enhance the learning on plants in elementary-level science.

The Huang Y. et al experiment was selected due to its characteristics: the use of a mobile device (PDA) and the GPS, and the educational objective of using technology to enhance the learning about geolocated elements (plants). However, an advanced activity can be created with QuesTInSitu. QuesTInSitu can be employed to include (1) assessment in situ and (2) to allow the monitoring of the activity in real time. We begin explaining the first improvement that we propose. As we have described in section 3, when a question is created by a teacher in QuesTInSitu this is associated to a geographical coordinate. In this sense, the MPLS and QuesTInSitu system can be combined to allow teachers to create and integrate assessment in situ activities in a Mobile Plant Learning experience. As described in the previous paragraph, the MPLS detects the position of the students through a GPS system and shows an e-map indicating the locations where the students have to go. Taking into account that the questions of QuesTInSitu have coordinates associated, then the questions also could appear in this e-map. Then the students can select a question (that automatically connects with the QuesTInSitu system) and answer it in situ. The students can participate more actively putting in 
practice their knowledge about the plants than when answering a paper-based test after the activity. Besides, since with QuesTInSitu the students can receive the feedback in situ, they can reflect on their knowledge in front of the plant. Moreover, students can associate their pictures or comments about botany to each specific question in the system.

The second contribution that QuesTInSitu can offer is a higher involvement of the teacher during the activity. Thanks to the monitoring service that QuesTInSitu incorporates, teachers can monitor in real time the position of their students and also know their progress (punctuations of the questions, the pictures and comments that they add). After doing the activity, the teacher can use the information saved in QuesTInSitu to discuss in class the answers selected by the students (e.g. the teacher can clarify the questions that have obtained more incorrect answers).

We claim that QuesTInSitu can be used to enhance a mobile-ubiquitous learning practice allowing the inclusion of assessment in situ activities. The routes activities are enriched with geolocated questions and provision of feedback in situ that can be combined with other learning resources. The architecture of the system enables its combination with other systems in order to share data such as: the coordinates of the QTI questions, the students' punctuations or pictures and comments added by the students. Moreover, QuesTInSitu supports the monitorization of the activity in real time.

\subsection{Example B: Facilitating the assessment in situ of the historical novel of Don Quixote}

The aim of the third scenario is to illustrate: how QuesTInSitu can be used by teachers to facilitate the edition and performance of existing educative itineraries with assessment purposes?

This scenario is based in existing best practices performed by teachers of literature courses in Spain. One of the books that are commonly read in Spanish literature courses is "Don Quixote". The story of the novel occurs in different real locations of Spain. In 2007 the routes cited in the Don Quixote novel were considered officially a European Cultural Itinerary (Cañizares, 2008). There exist a lot of educative materials on the web that relates the events of the novel with the real itinerary (e.g.

http://www.quijote.tv/escuela1.htmin Spanish). However, it can be a hard task for the teachers to relate these contents with their associated location in order to perform and assessment in situ activity with their students. In this scenario we explain how QuesTInSitu could be used to facilitate the creation of an assessment in situ activity about the Don Quixote book for a Spanish literature subject.

QuesTInSitu enables teachers to design an assessment in situ route that can contain part of (or all) the different places of the official itinerary that are cited in the novel without going physically to the different emplacements. The QuesTInSitu's GUI facilitates the web map edition of the zone selected. For each important location of the itinerary, the system offers functionalities that enable the creation and geolocation of questions that have a relation with the facts described in the book. Once the map with the test-route is created, the students will have the possibility of doing physically the itinerary with a mobile phone. When the students will be in front of a location selected by the teachers as a questionpoint, the students will have to answer the related question using QuesTInSitu. The question will be automatically corrected and the students will be able of reflecting and comparing their response with the correct answer observing the environment. After answering a question, the students will have the possibility of uploading a picture or/and a comment of each location to the application using the mobile phone. In order to assess the students, the teachers have to check the information associated to each student in QuesTInSitu. Teachers can monitor and review the score obtained in each question and also they can evaluate the pictures and comments uploaded by the students.

The proposed scenario would help teachers to create an ubiquitous assessment in situ activity related with the existing Don Quixote cultural itinerary and also to motivate their students in the learning of the Don Quixote book.

\section{Discussion}

The scenarios analysed in previous sections have been used to understand the benefits and limitations of using QuesTInSitu for doing assessment in situ activities. The scenarios provide further indications that can be used to confirm the statement presented at the beginning of section 4 and to provide evidences that can be used to answer the research questions formulated in sections 4.1.2, 5.1 and 5.2. The results obtained in the main scenario, Discovering Barcelona, are supported with the analysis done in examples A and B. This final analysis is used to evaluate whether the objectives formulated in section 4.1.2 are achieved. 
In order to conclude the discussion, we also discuss the advantages and limitations of using GPS data and LBS, as implemented in QuesTInSitu, versus others technologies such as QR-Codes or NFCs to do assessment in situ activities.

\subsection{Discussion and Analysis of the three scenarios using QuesTInSitu}

We can state that QuesTInSitu can adequately support the execution of real assessment in situ activities providing educational benefits. The results of the scenarios show how QuesTInSitu is useful for giving support in situ during the realization of an assessment in situ activity.

The strategy of giving in real time and in situ the feedback and the score is an essential aspect that helps students to understand better the content of the questions of the test-route. The first result of the first scenario, Discovering Barcelona, shows how students value positively the provision of feedback, and the second scenario illustrates how a ubiquitous experience can be enriched by including automatic assessment in situ.

During the realization of an assessment in situ activity students achieve and practice specific knowledge and skills regarding the topic selected by the teachers and it also promotes other transversal skills such as communication, teamwork or spatial orientation (see the second result of the Discovering Barcelona scenario).

From the point of view of the teachers the monitoring service allows them to understand in real time what the students are doing and their progress. The teachers of the first scenario, value this functionality as one of the most useful for them because it makes possible not to go with the students and they can focus their attention on checking the students' evolution on the test-route. Moreover the authoring functionalities of QuesTInSitu have been designed with the objective of facilitating the creation of assessment in situ activities. The third scenario, example B, exemplifies how teachers can take profit of these functionalities to design assessment in situ activities.

The results confirm that the combination of the QuesTInSitu applications: the assessment engine (NewAPIS), the LBS (e.g. Mscape or the MPLS) and the web map service (Google Maps) through devices such as computers and mobile phones with GPS and Internet enhances the correct realisation of an assessment in situ activity. Mediating the visualization of questions with a LBS is not essential, but the geolocation information helps to provide dynamism to the activity and to create a more accurate context-aware activity. This result opens the possibility of using QuesTInSitu with other ubiquitous-learning systems, as the example A proposes.

Finally, the data collected demonstrate that the structure of the activity had a positive direct impact in the students' motivation (see the third result of the Discovering Barcelona scenario). The students indicated that the use of the systems and devices employed during the activity help and motivate them on its correct performance. The use of technology enables to perform the activity without the physical presence of the teachers. The results indicate that this fact makes that students participate actively in the activity. As a consequence they are more motivated and concentrated in the tasks of answering the questions. This result agree with the analysis performed by the Futurelab (Naismith et al., 2004) about the Savannah project of Facer et al. (2004) (see Section 2.2 where the project is briefly explained). A quotation extracted from the report explains: "When the teacher took control, the students became more passive and resistant to engagement. To be successful as a learning experience, the game (we add "the activity") needs to allow the students to control their own learning."

\subsection{Discussion: understanding the use of smartphones in assessment in situ activities}

As has been explained, QuesTInSitu is a system that combines the use of smartphones, an assessment engine and a LBS (see Figure 5). Questions appear when students are physically located in the correct location, using the GPS service and the Internet connection of the smartphone. However, other combination of technologies can be used to carry out an assessment in situ activity. As we have seen in the literature review section of this paper, other authors have used technologies such as NFC-RFID tags or tour-based applications (Huang, Y. M., 2009; Hwang, G-J., 2011; Pérez-Sanagustín, M., accepted) to create location-based activities. Other technologies such as QR-Codes also can be used to extend the information of a real object (Rohs \& Gfeller, 2004; Susono \& Shimomura, 2006). The experiment and scenarios presented in this paper, and the related experiments, allow us to discuss the advantages and limitations of using smartphones with GPS and Internet connection compared with other technologies for the creation of assessment in situ activities. This section is devoted to provide criteria about which technology is the most adequate considering the physical characteristics of the assessment environment: 
Open spaces: when the assessment environment is an open space with GPS/Internet coverage (e.g. a district of a city or a park) the use of smartphones using a system like QuesTInSitu is more adequate than other technologies because: (1) Teachers do not need to interact physically with the environment, putting manually NFC-RFIDs or QR-Codes tags in the associated places. Then, teachers avoid the need of checking the space, or having it physically under control. (2) When the questions do not depend of a physical element (like an augmented card) and they are associated to real coordinates, the flow of questions can be easily modified, even during the performance of the assessment activity (using a computer). (3) Questions are associated to real coordinates, and the real location of the student is known through the information send by the GPS of the smartphone to the system. The system can detect where the users are and adapt the activity considering their positions. (4) As has been analysed in the previous section, students indicated that the automatic appearance of questions confirms that they are correctly located. However, when students have to select manually the questions, for instance reading a QR-Code located in a map or using a tour-based application, there exist the risk of being situated incorrectly.(5) Again, as mentioned by the students during the experiment, the automatic visualization of questions (controlled by the GPS signal) gives dynamism to the process of reading/answering questions. This automation get students more "excited" and motivates them to perform the activity.(6) The Internet connection allows knowing in real time the students' position, It can be used to monitor, in real time the progress (locations, scores achieved) of the students in the activity.(7) When the distance between question has to be minimal it is better to do not use GPS coordinates to control the questions. However, when questions can be separated, it is desirable to leave at least a distance of 10 meters between each question/test in order to give the opportunity to the students to feel that they have to walk for finding new questions.

Closed spaces (or small / controlled open spaces): when an assessment activity is carried out in a space like a museum or a campus of a university it is very difficult or impossible to do an assessment activity with GPS signal. Then, QuesTInSitu cannot be used with automatic visualization of questions. In these cases, it is most adequate using other solutions such as RFID tags, QR-Codes or tour-guide applications: (1) Teachers will have to put manually the tags in the corresponding locations or prepare a map of the zone with the corresponding codes indicated. Considering that the space is closed having under control the contextual-material is not very risky. (2) When the Internet connection is bad or null, due the characteristics of the space, all the material (tests, images, audios) of the assessment activity has to be saved in the memory of the mobile device. This fact increases the tasks that have to be done by the teacher before the execution of the assessment activity. (3) In addition, in these situations the teacher cannot make changes during the activity or monitor it in real time. (4) The responsibility of reading correctly the questions fall on the students, they have to do the action: scan the QR-code, read a map or compare the information of the guide-tour with the real word. (5) When students are in closed spaces using a smartphone to perform an assessment activity, knowing their real position is not possible, then it is very difficult to send feedback or hints to guide them during the route.

Finally, the re-usability and adaption of the assessment material become easier in the first case than in the second case because the dependence of physical objects like (cards, codes, maps) difficult these tasks. In addition, the use of educational technology standards, like QTI in QuesTInSitu, facilitates the interoperability of learning materials or the outputs of the system (e.g., scores) with other compliant systems.

\section{Conclusions and Future work}

Despite the large number of Computer-based Testing solutions in use nowadays, there is still the need of creating innovative ways of representing tests (Boyle \& Hutchison, 2009; Elliott, 2008; Parshall et al., 2010; Ridgway \& McCusker, 2003). This paper discusses how technologies, especially smartphones, can be employed to assess the students' knowledge and skills (Dearnley et al., 2009). The term Computingbased Testing (CBT) is proposed to represent better the use of technology to implement test-activities. Three representative m-test tools (de-Marcos et al., 2010; Triantafillou et al., 2008; Zhang et al., 2010) are analyzed with the objective of understanding the advantages and limitations of the current approaches. The highlighted benefit is that using mobiles phones students can practice self-assessment activities anytime and anywhere. However we claim that the limitation of these solutions is that they do not exploit all the benefits that mobile phones and related technologies have to assess the students' knowledge and skills. Moreover, these solutions do not contribute in terms of the activity itself. The mobile phone is presented as a medium that facilitates the answering of a test wherever the user wants, and not as a 
medium that can provide other ways of implementing assessment activities based on tests. The paper has also presented other m-learning experiences, with (and without) assessment purposes, where features such as the camera or the GPS are important factors that contribute to the success of the activity (Facer et al., 2004; Huang et al., 2009; Sharples et al., 2002; Hwang, G-J., 2011). As an example, the Mscape system (Stenton et al., 2007) is presented in detail. This system allows authors to implement ubiquitous mlearning activities where being physically in an open environment is essential for the correct performance of the activity.

The state of the art has led us to identify the need of exploiting the possibilities that mobiles devices provide for designing new types of assessment activities based on tests. As a step forward, this paper presents an innovative CBT activity: Assessment in situ; which considers the use of mobile phones for answering questions that are dependent of a location. This means that the questions are related with a geographical coordinate and students have to be located in the correct position in order to answer the question. Assessment in situ activities offer a new way of using and representing tests: they are conceived as educational routes that serve assessment purposes. The QuesTInSitu system enables the creation of these enriched test-based routes. The questions are grouped in a test-route using a webmap service. The questions follow the QTI standard (IMS, 2006). It has been selected because its data model helps us to modellate the assessment resources (questions, tests and results reports).

Three scenarios, an experiment implementing a real assessment in situ activity and two illustrative examples (the extension of a related research scenario and the enrichment of a scenario based on real practices) have been presented in order to evaluate the benefits of QuesTInSitu. The results obtained help us to state that QuesTInSitu provides adequate support for teachers and students during the realisation of an assessment in situ activity. It allows teachers the possibility of creating enriched and motivating ubiquitous m-test activities that facilitates the assessment and the development of specific and transversal students' knowledge and skills adapted to diverse educational disciplines (e.g. Geography and History, Botany or Literature).

The use of a Location-Based System in the experiment has enabled us to study the advantages of the automatic visualization of questions. Although the results obtained demonstrate that the LBS is not essential for the correct development of an assessment in situ activity, both students and teachers value positively that the questions were visualized when the students were well located in the correct position. In order to understand better the advantages and limitations of using GPS and Internet connection to carry out an assessment in situ activity, the paper compares this technology with other contextual-based technologies. The discussion concludes saying that in open spaces with Internet connection the use of geolocated data allows teachers to know the position of their students, and manage the assessment information online and in real time. The use of a LBS or GPS enables the performance of a more accurate assessment in situ activity.

As future work we plan to study deeper which type of geolocated information can be useful to provide support to students during the performance of an assessment in situ activity. Moreover, considering the teachers' comments, the communication mechanisms between the teachers and the students during the activity have to be enhanced. The current version of QuesTInSitu does not enable teachers to send text, audio or video messages to their students during the activity. Our plans include developing a mechanism that will enhance the communication and socialization between participants in real time. We also plan to add more types of questions to be used in the test-routes of QuesTInSitu. Last but not least, efforts will be also devoted to perform more experiments in different educational disciplines.

As a reflection we conclude with the vision of the JISC report "Effective assessment in a digital age" which indicates that the assessment of the future has to provide greater opportunities for creating enriched and authentic assessment experiences making use of personal devices (JISC, 2010). We claim that it is not necessary to wait to the future because the Assessment in situ activity presented in this paper fits the characteristics mentioned by the JISC. Answering traditional types of questions in situ with mobiles making use of location-based features while students follow a route enables the creation of an enriched and authentic CBT experience. It engages students in the task of answering a test and provides a new way of using tests that teachers can use to assess higher order skills.

\section{References}

Blat, J., Navarrete, T., Moghnieh, A. \& Batlle-Delgado, H. (2007). A QTI Management System for Service Oriented Architectures. Proceedings of the TENCompetence Open Workshop on Service Oriented Approaches and Lifelong Competence Development Infrastructures (pp. 175-181). Manchester, UK.

Boyle, A. \& Hutchison, D. (2009). Sophisticated tasks in e-assessment: What are they and what are their benefits? Assessment \& Evaluation in Higher Education, 34(3), 305-319.

Bull, J., \& McKenna, C. (2004). Blueprint for computer-assisted assessment. London, U.K.: RoutledgeFalmer. 
Cañizares, M. C. (2008). La "ruta de don quijote" en castilla la mancha (españa): Nuevo itinerario cultural europeo. Nimbus: Revista de climatología, meteorología y paisaje, ISSN 1139-7136, No 21-22, 55-75 (in Spanish). Available fromhttp://dialnet.unirioja.es/servlet/articulo?codigo=2899589 (accessed Dec, 2010).

Chen, C. H. (2010). The implementation and evaluation of a mobile self- and peer-assessment system. Computers \& Education, 55(1), 229-236.

Dearnley, C., Taylor, J., Hennessy, S., Parks, M., Coates, C., Haigh, J., et al. (2009). Using mobile technologies for assessment and learning in practice settings: Outcomes of five case studies. International Journal on ELearning, 8(2), 193-207.

De-Marcos, L., Hilera, J. R., Barchino, R., Jímenez, L., Martínez, J. J., Gutiérrez, J. A., et al. (2010). An experiment for improving students performance in secondary and tertiary education by means of m-learning autoassessment. Computers \& Education, 55(3), 1069-1079.

Elliott, B. M. (2008). Modernising assessment in the age of web 2.0. Scotland: Scottish Qualifications Authority. Available from http://www.scribd.com/doc/461041/Assessment-20 (accessed Dec, 2010).

Facer, K., Joiner, R., Stanton, D., Reid, J., Hull, R., \& Kirk, D. (2004). Savannah: Mobile gaming and learning? Journal of Computer Assisted Learning, 20(6), 399-409.

Harchay, A., Cheniti, L., \& Braham, R. (2010).An investigation of the enhancement and the formal description of IMS/QTI specification for programming courses. In Proceedings of the 2010 10th IEEE International Conference on Advanced Learning Technologies(pp. 113-115).Sousse, Tunisia, 5-7 July, 2010.

Huang, Y. M., Lin, Y. T., \& Cheng, S. C. (2009). Effectiveness of a mobile plant learning system in a science curriculum in taiwanese elementary education. Computers \& Education, 54(1), 47-58.

Hwang, G., \& Chang, H. (2011). A formative assessment-based mobile learning approach to improving the learning attitudes and achievements of students.Computers \& Education, 56, 1023-1031.

IMS. (2006). IMS question \& test interoperability specification v2.0/v2.1. Available from http://www.imsglobal.org/question/index.html (accessed Dec, 2010).

JISC. (2010). Effective assessment in a digital age. Bristol, UK. Available from http://www.jisc.ac.uk/digiassess (accessed Dec, 2010).

Jones V. \& Jo H. J. (2004). Ubiquitous learning environment: an adaptive teaching system using ubiquitous technology. In Proceedings of the 21st ASCILITE conference (pp. 468-474), Perth, Western Australia, 5-8 December, 2004.

Lincoln, Y. S., \& Guba, E. G. (1985). Naturalistic inquiry. Beberly Hills, California: Sage.

Mackay, W. (1998). Augmented reality: Linking real and virtual worlds: A new paradigm for interacting with computers. In Proceedings of the Working Conference on Advanced Visual Interfaces (pp.13-21), L'Aquila, Italy, 24-27 May, 1998.

Martinez, A., Dimitriadis, Y., Rubia, B., Gomez, E., \& de la Fuente, P. (2003). Combining qualitative evaluation and social network analysis for the study of classroom social interactions. Computers \& Education, 41(4), 353-368.

Miao, Y., Tattersall, C., Schoonenboom, J., Stevanov, K., \& Aleksieva-Petrova, A. (2007). Using open technical elearning standards and service-orientation to support new forms of e-assessment. In Proceedings of the Second TENCompetence Open Workshop on Service Oriented Approaches and Lifelong Competence Development Infrastructures (pp. 183-190), Manchester, United Kingdom, 11-12 January, 2007.

Naismith, L., Lonsdale, P., Vavoula, G., \& Sharples, M. (2004). Literature review in mobile technologies and learning No. 11. University of Birmingham: Futurelab.

Nova, N., Girardin, F., \& Dillenbourg, P. (2005). 'Location is not enough!': An empirical study of locationawareness in mobile collaboration.In Proceedings of the IEEE International Workshop on Wireless and Mobile Technologies in Education (WMTE '05) (pp. 21-28),Tokushima, Japan, 28-30 November, 2005.

Parshall, C. G., Harmes, J. C., Davey, T., \& Pashley, P. (2010). Innovative items for computerized testing.In van der Linden, W. J., \& C. A. W. Glas (Eds.), Elements of Adapting Testing, (pp. 215- 230). Norwell, MA: Kluwer Academic Publishers.

Pérez-Sanagustín, M., Ramírez-González, G., Hernández-Leo, D., Muñoz-Organiero, M., Santos, P., Blat, J., et al. (accepted). Discovering the campus together: A mobile and computer-based learning experience.Journal of Network and Computer Applications, JNCA.

Pintrich, P. R. (2003). A motivational science perspective on the role of student motivation in learning and teaching contexts. Journal of Educational Psychology, 95(4), 667-686.

RQuestionMark (2010). Questionmark case study: Mobile assessments at eastern washington University mobile assessments at washington university. Available from http://www.questionmark.com/us/casestudies/ewu pda.aspx (accessed Dec, 2010).

Ridgway, J., \& McCusker, S. (2003). Using computers to assess new educational goals. Assessment in Education, 10(3), 309-328.

Rohs, M., \& Gfeller, B. (2004). Using camera-equipped mobile phones for interacting with real-world objects. Proceedings of Advances in Pervasive Computing, 265-271.

Schuler, D., \& Namioka, A. (Eds.). (1993). Participatory Design: Principles and Practices. Lawrence Erlbaum, HiIlsdale, N.J.

Sharples, M., Corlett, D., \& Westmancott, O. (2002). The design and implementation of a mobile learning resource. Personal and Ubiquitous Computing, 6(3), 220-234.

Stenton, S. P., Hull, R., Goddi, P. M., Reid, J., Clayton, B., Melamed, T., et al. (2007). Mediascapes: Context-aware multimedia experiences. In IEEE Multimedia, 14(3), 98-105.

Susono, H., \& Shimomura, S. (2006). Using mobile phones and QR codes for formative class assessment. Current Developments in Technology-Assisted Education. Badajoz, Spain: FORMATEX. 1006-1010. 
Triantafillou, E., Georgiadou, E., \& Economides, A. A. (2008). The design and evaluation of a computerized adaptive test on mobile devices. Computers \& Education, 50(4), 1319-1330.

Wegerif, R. (2002). Literature Review in Thinking skills, Technology and Learning. School of Education, Open University: NESTA Futurelab. Available from http://www.futurelab.org.uk/resources/documents/lit_reviews/Thinking_Skills_Review.pdf $\quad$ (accessed Dec, 2010).

Zhang, P., Wills, G., Gilbert, L., Warburton, B., \& Bacigalupo, D. (2010). IMS QTI engine on android to support offline mobile learning. In Proceedings of the Computer Assisted Assessment Conference, Southampton, U.K., 20-21 July, 2010. 